\title{
Efectos de la infección por COVID-19 en pacientes renales. Encuesta Latinoamericana ACECANH 2020
}

\author{
Karen Courville ${ }^{1,2,3 *}$, Florencio McCarthy ${ }^{2,4}$ y Régulo Valdés ${ }^{2,5}$ \\ ${ }^{1}$ Departamento de Nefrología, Hospital "Dr. Gustavo N. Collado,"Chitré; ${ }^{2}$ Asociación Centroamericana y del Caribe de Nefrología e Hipertensión (ACECANH); \\ ${ }^{3}$ Departamento de Investigación, Instituto de Ciencias Médicas, Las Tablas, Los Santos; ${ }^{4}$ Servicio de Nefrología, Hospital del Niño Dr. José Renán Esquivel; \\ ${ }^{5}$ Servicio de Nefrología, Hospital Dr. Arnulfo Arias Madrid, Coordinación Nacional de Diálisis, Caja de Seguro Social. Panamá
}

\section{Resumen}

Introducción: La epidemia de COVID ha ocasionado un cambio en los procesos de atención de los pacientes crónicos. Objetivo: La Asociación Centroamericana y del Caribe de Nefrología e Hipertensión (ACECANH) ha evaluado la forma en que se ha afectado el cuidado de los pacientes renales en Latinoamérica. Material y métodos: Se realizó una encuesta virtual para valorar los cambios de actividad relacionados con la asistencia, el seguimiento y el diagnóstico de los pacientes con enfermedad renal crónica en diálisis. Resultados: Se evaluaron 137 encuestas, con participación del 41\% de Centroamérica, 37\% de Sudamérica; $17 \%$ del Caribe; y 5\% de Norteamérica. Sesenta y ocho por ciento refirió afectación en la atención en consulta de enfermedad renal crónica; en un 54\% de los centros, los pacientes no han acudido a su tratamiento habitual de hemodiálisis; $40 \%$ ha tenido dificultades por falta de medicamentos o insumos; $y$ 36\% indicó dificultades en el suministro de fármacos inmunosupresores en el trasplante renal. Conclusiones: Deben continuarse las medidas de prevención de infección y el uso de equipos de protección personal adecuados, pero es preciso hacer los mejores esfuerzos como comunidad nefrológica para vigilar que las instituciones proporcionen los tratamientos necesarios y no se interrumpa la atención de los pacientes renales.

Palabras claves: COVID-19. América Latina. Nefrología. Encuesta. Paciente. Enfermedad renal crónica.

Impact of COVID-19 infection in renal patients. 2020 ACECANH Latin American Survey

\section{Abstract}

Introduction: The COVID epidemic has led to a change in chronic patient care processes. Objective: The Central American and Caribbean Association of Nephrology and Hypertension (ACECANH) has wanted to assess how the care of renal patients in Latin America has been affected. Material and methods: A virtual survey was conducted to evaluate activity changes related to the attendance, monitoring and diagnosis of patients with Chronic Renal Disease in dialysis. Results: 137 surveys were evaluated, with participation of $41 \%$ from Central America; $37 \%$ of South America; $17 \%$ of the Caribbean; $5 \%$ of North America. Sixty-eight percent reported involvement in care in consultation of CKD; in $54 \%$ of the centres, patients have had to miss their usual haemodialysis treatment; $40 \%$ have struggled due to lack of medicines or supplies; $36 \%$ reported difficulties in the supply of immunosuppressive drugs in renal transplantation. Conclusions: Infection prevention measures and

\footnotetext{
Correspondencia:

*Karen Courville

E-mail: kavac7@gmail.com

Fecha de recepción: 28-08-2020

Fecha de aceptación: 31-10-2020

DOI: $10.24875 /$ NEFRO.200000461 2444-9032/@ 2020 Sociedad Latinoamericana de Nefrología e Hipertensión. Publicado por Permanyer. Este es un artículo open access bajo la licencia CC BY-NC-ND (http://creativecommons.org/licenses/by-nc-nd/4.0/).

Disponible en internet: 17-12-2020

Nefro Latinoam. 2020;17:81-85 www.nefrologialatinoamericana.com
} 
the use of appropriate personal protective equipment should be continued, but we must make our best efforts as a nephrological community to ensure that our institutions provide the necessary treatments so that kidney care is not interrupted.

Key words: COVID-19. Latin America. Nephrology. Survey. Patient. Chronic Kidney Disease.

\section{Introducción}

La pandemia del COVID-19 es una infección que se ha transformado en un corto tiempo en la enfermedad más importante para todos los sistemas de salud y los gobiernos de todos los países del mundo. Es la única afección que ha orillado al confinamiento domiciliario, con restricciones de movilización y cambios en todas las actividades de la vida diaria.

En la atención médica ha producido un drástico cambio en los procesos de atención y llevado al cierre de las consultas externas, lo cual ha reducido la atención de los pacientes crónicos y el seguimiento de sus comorbilidades porque la mayor parte de los esfuerzos ha debido enfocarse en la atención en cuartos de urgencias, en particular de los pacientes con síntomas respiratorios, y en unidades de cuidados críticos por las complicaciones que produce este virus.

En Latinoamérica, la situación cambió de forma notable de unas cuantas defunciones al 30 marzo de 2020 a más de 100,000 al 30 de julio, ${ }^{1,2}$. Los sistemas de salud pasaron de la preparación a la desesperación y al agotamiento del personal; más aún, los sistemas de salud resultaron deficientes e insuficientes, con múltiples problemas sociales y económicos ${ }^{3}$. La Asociación Centroamericana y del Caribe de Nefrología e Hipertensión (ACECANH) ha evaluado la manera en que ha continuado el cuidado de los pacientes renales sometidos a diálisis en Latinoamérica durante esta pandemia, para lo cual utilizó una encuesta dirigida a los especialistas en nefrología en sus diferentes centros de atención.

\section{Métodos}

Se realizó una encuesta virtual por medio del boletín mensual enviado por correo electrónico a los nefrólogos suscritos a la página web www.acecanh.org. Se publicó el 20 de julio de 2020 y se mantuvo disponible hasta el 30 de julio de 2020. La encuesta se realizó con la herramienta en línea SurveyMonkey. El contenido de la encuesta se resume en la figura 1. La región de ACECANH comprende a ocho sociedades de nefrología con 191 nefrólogos adscritos. Debido al alcance de la modalidad virtual, un $60 \%$ de los visitantes de la página web procede de Centroamérica y el Caribe, pero además participó un $40 \%$ de visitantes de Sudamérica y Norteamérica.

Se formularon 27 preguntas divididas en tres partes: a) datos generales e informativos sobre los participantes y las actividades que realizan en nefrología; b) cambios de actividad relacionados con la atención de los pacientes con enfermedad renal crónica (ERC) 5 y diálisis; y c) la evaluación de cambios relacionados con complicaciones por la falta de algunos tratamientos médicos en pacientes con ERC 5 y trasplante renal. Los datos se tabularon y graficaron entre el 31 de julio y el 5 de agosto.

\section{Resultados}

Se recibieron 137 encuestas en los 10 días habilitados. El $98 \%$ de los participantes trabaja en el área de nefrología. El 92\% corresponde a médicos nefrólogos, $5 \%$ a médicos generales que colaboran en nefrología, $2 \%$ a personal de enfermería y $1 \%$ a otro personal de salud. Los encuestados eran hombres en una proporción de $64 \%$. El $20 \%$ lo integraron personas de 20 a 40 años, el $53 \%$ individuos de 41 a 60 años y el $26 \%$ mayores de 61 años.

En cuanto a la región de procedencia en el continente, el $41 \%$ provenía de Centroamérica, $37 \%$ de Sudamérica, $17 \%$ del Caribe y $5 \%$ de Norteamérica. Al momento de la encuesta, el $78 \%$ de los encuestados trabajaba en un hospital que atendía a pacientes positivos a COVID. El 90\% trabajaba en unidades de hemodiálisis y $56 \%$ en programas de diálisis peritoneal. Al preguntar sobre el número de pacientes en las diferentes modalidades de diálisis que desarrolló infección por COVID-19, la mayor cantidad se hallaba en hemodiálisis, seguida de diálisis peritoneal y, en menor cantidad, enfermos con trasplante renal (Fig. 2). El 54\% de los encuestados señaló que había evaluado a pacientes con lesión renal aguda relacionada con COVID.

Hasta 54\% notificó inasistencias a sesiones de hemodiálisis debido a la infección por COVID-19 (Fig. 3), mientras que el porcentaje de incumplimiento del tratamiento de diálisis peritoneal en casa fue menor del 2\% (Fig. 4). El 68\% de los encuestados comunicó una interrupción de la atención en consulta de diálisis y $46 \%$ un aumento de los pacientes con diagnóstico de ERC 5 de novo con requerimiento de tratamiento de reemplazo renal (TRR). 


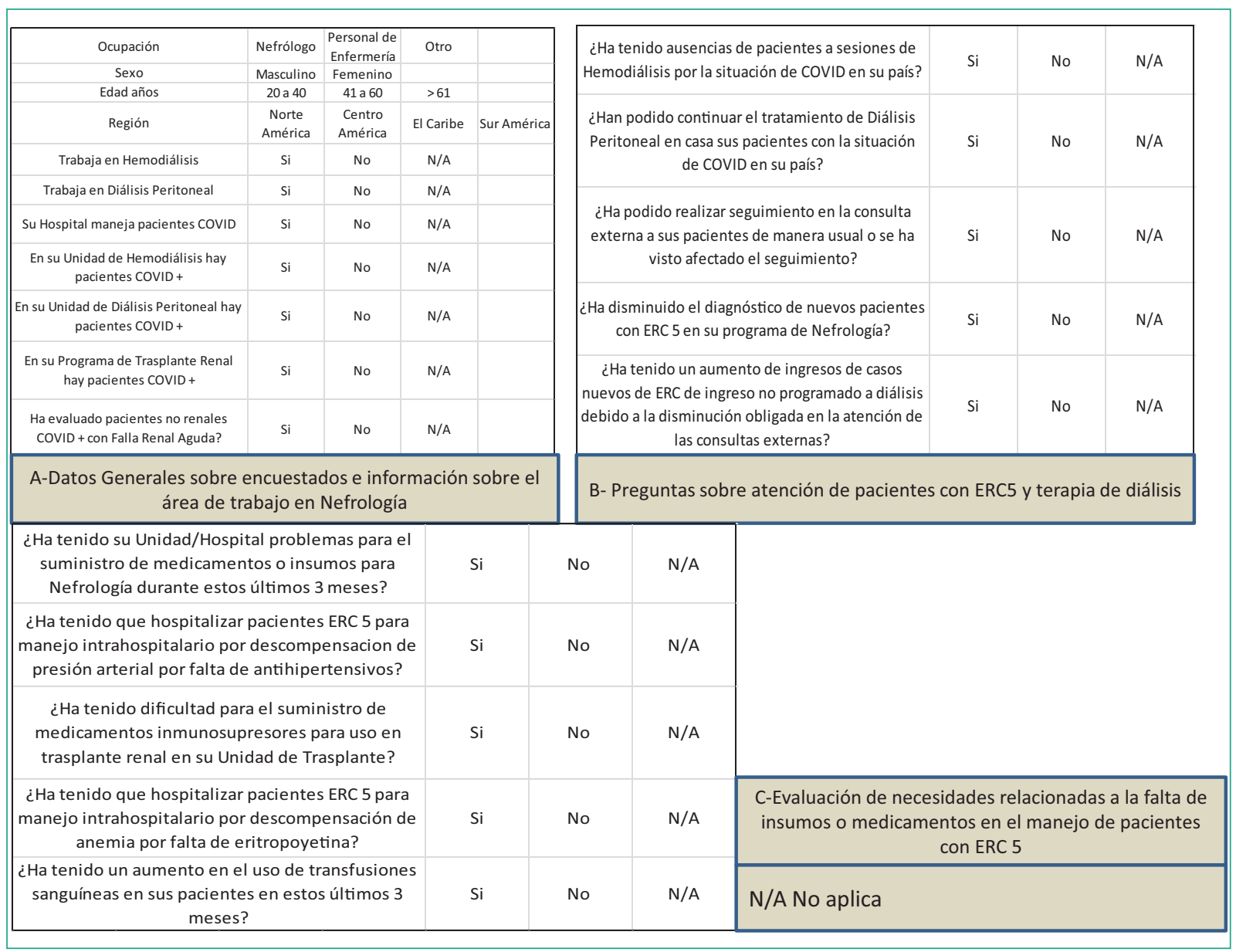

Figura 1. Preguntas formuladas en la encuesta en línea.

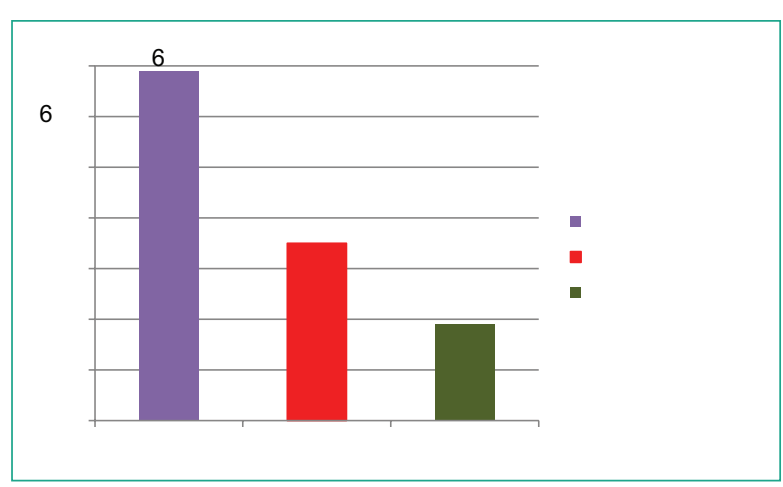

Figura 2. Diagnóstico de pacientes positivos a COVID-19 en las diferentes modalidades de tratamiento de reemplazo renal.

disponibilidad de acceso a la salud ${ }^{4}$, factores que pueden agravar a los pacientes con enfermedades crónicas.

Los pacientes de hemodiálisis deben trasladarse a su centro de tratamiento tres veces a la semana: esto
Cuarenta por ciento de los centros ha tenido dificultades por falta de medicamentos o insumos de nefrología en los últimos tres meses y $36 \%$ en el suministro de fármacos inmunosupresores en el trasplante renal.

De manera más específica, en la evaluación de las complicaciones por falta de medicamentos, un $18 \%$ había requerido hospitalizar a pacientes para controlar las crisis hipertensivas por falta de antihipertensivos y un $8.8 \%$ había hospitalizado a pacientes para tratar la anemia por falta de fármacos eritropoyéticos. Durante los últimos tres meses, un $15 \%$ de los encuestados informó un mayor número de transfusiones sanguíneas para el tratamiento de la anemia.

\section{Discusión}

Los efectos de la infección por COVID-19 son un tema complejo que puede traer consecuencias importantes, en virtud del aumento del desempleo, la pobreza y la 


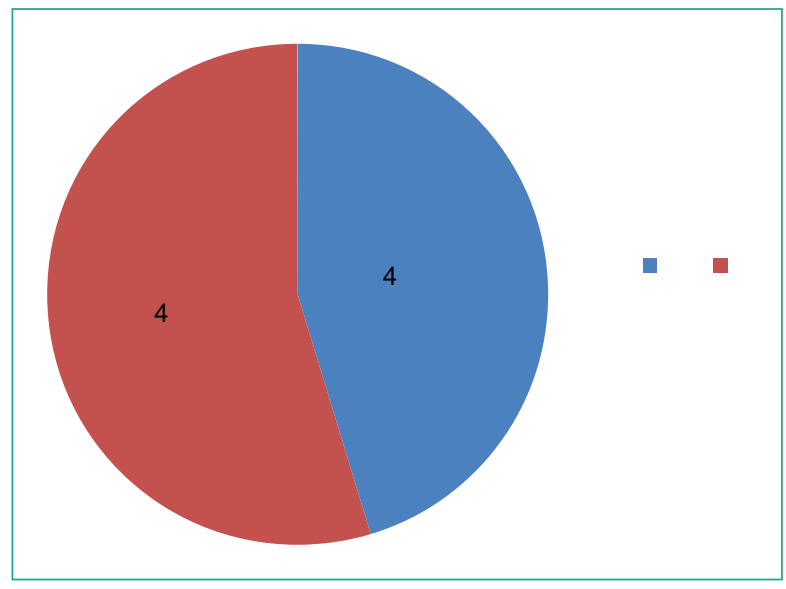

Figura 3. Evaluación de ausencias de pacientes con ERC 5 para tratamiento de hemodiálisis.

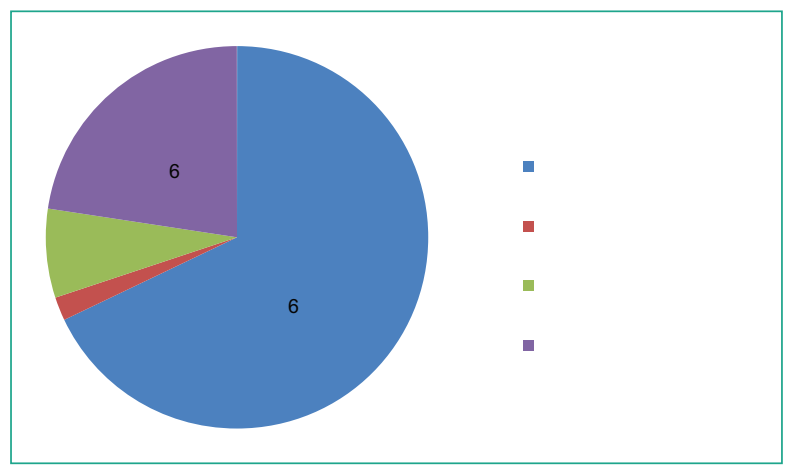

Figura 4. Porcentaje de cumplimiento de diálisis peritoneal en casa durante la pandemia.

eleva su riesgo de exposición al SARS-Cov-2. México tiene una prevalencia de $12.2 \%$ de enfermedad renal crónica ${ }^{5}$, con más de 55,000 pacientes bajo tratamiento de reemplazo renal ${ }^{6}$ que deben desplazarse todas las semanas. La prevalencia de hemodiálisis en Latinoamérica es de 442 pmp (por millón de personas), según el último Registro Latinoamericano de Diálisis y Trasplante ${ }^{7}$. Los resultados de esta encuesta demuestran que en un $54 \%$ de los centros, los pacientes han debido faltar a su tratamiento usual de hemodiálisis por motivos relacionados con la pandemia por COVID-19. La paralización o disminución de los medios de transporte, las cuarentenas obligadas, la escasez de recursos económicos por el aumento del desempleo y el miedo a la infección incrementan el riesgo de que los pacientes bajo hemodiálisis no acudan a todas 0 alguna de sus sesiones programadas de diálisis, lo que eleva el riesgo de complicaciones vinculadas con la falta de diálisis. Con las nuevas opciones de telesalud para atender afecciones crónicas, la hemodiálisis no puede cambiar su modalidad de "tratamiento en centro" y el personal de salud debe continuar su trabajo sin distanciamiento físico.

En España, la prevalencia de COVID-19 en pacientes bajo tratamiento de reemplazo renal ha sido de $2.8 \%$, lo que representa una cifra aproximada de 1,700 pacientes. El $64 \%$ de estos enfermos recibe hemodiálisis, $32 \%$ es objeto de trasplante y $3.2 \%$ se halla bajo diálisis peritoneal ${ }^{8}$. Los resultados en Latinoamérica se asemejan a los de España, en donde predominan los pacientes infectados por COVID en hemodiálisis debido a que no es posible cambiar la modalidad de "tratamiento en centro" y los pacientes no pueden evitar el traslado a sus unidades dos a tres veces por semana.

En cuanto a la atención de los pacientes, muchos hospitales han cancelado las consultas externas para impedir los desplazamientos de los pacientes y las aglomeraciones en las salas de espera para reducir el riesgo de contacto del personal ${ }^{9}$. Un $68 \%$ de los encuestados respondió que el seguimiento de los pacientes se ha afectado y sólo un $27 \%$ ha continuado la atención de la manera habitual. La omisión de las citas de atención de morbilidad puede tener efectos contraproducentes en la salud de la población y, tras el transcurso de algunos meses, debe considerarse la forma de reorganizar la atención de los pacientes.

La atención de las autoridades se ha enfocado en comprar equipos de protección personal, aseo y medicamentos para tratar la infección por COVID y en muchos hospitales se han adecuado nuevas áreas para tratamiento de pacientes infectados, lo que representa altos costos no presupuestados. Un $40 \%$ de los encuestados refirió deficiencias en suministros de fármacos o insumos de nefrología en sus unidades u hospitales, como antihipertensivos e inmunosupresores para trasplante renal; esto pone en riesgo inmediato la sobrevida del injerto y el paciente, por lo que no debe perder su carácter prioritario, incluso en tiempos de pandemia.

La Organización Panamericana de la Salud (OPS) ha informado la considerable disminución de la disponibilidad de sangre debido a la reducción de las donaciones voluntarias, de tal modo que se han agotado las reservas de los bancos de sangre de los centros hospitalarios $^{10}$. La menor disponibilidad de agentes eritropoyéticos ${ }^{11}$ puede incrementar el requerimiento de transfusiones sanguíneas en la población con ERC 5 
en diálisis, por lo que es necesario asegurar que los pacientes reciban sus medicamentos de manera regular para evitar las complicaciones cardiovasculares de la anemia crónica ${ }^{12}$.

Debido al cierre de los hospitales de día y consulta ambulatoria, muchos pacientes con enfermedad crónica avanzada dejaron de consultar o perdieron citas con médicos especialistas, lo que ha producido un aumento de los casos con ERC que acuden para inicio de TRR no programado, según se indicó en $46 \%$ de esta encuesta; esto podría acentuar la crisis de falta de riñones artificiales que ya existe en muchos países de Latinoamérica ${ }^{7}$.

Se han establecido recomendaciones internacionales $^{13}$ y algunos países han creado sus propias guías nacionales, orientadas al tratamiento y prevención de las complicaciones de la infección por COVID. En los pacientes bajo TRR es todavía importante aplicar medidas de educación, detección, seguimiento y aislamiento en las unidades.

Esta encuesta tiene algunas limitaciones. La encuesta virtual tuvo una duración de 10 días en el boletín, período en el que los nefrólogos que contestaron fueron los más identificados con el tema, los más colaboradores o los más interesados en temas de investigación, por lo que existe un sesgo en la obtención de los resultados.

\section{Conclusiones}

Los datos recolectados muestran la información sobre los primeros meses de la pandemia. Los pacientes en hemodiálisis son los más afectados, en comparación con los pacientes bajo diálisis peritoneal; las restricciones de movilización y la interrupción de la atención de los pacientes con enfermedad renal crónica han producido un incremento del número de pacientes para inicio no programado de diálisis. Deben considerarse medidas como la promoción del tratamiento de diálisis peritoneal para los pacientes con ERC 5. Es preciso continuar con las medidas de prevención de infección y el uso de equipo de protección personal adecuado para todo el personal y los pacientes en todas las modalidades terapéuticas.

\section{Financiamiento}

Este trabajo recibió financiamiento de ACECANH.

\section{Agradecimientos}

Los autores agradecen a todos los colegas que colaboraron con las respuestas de la encuesta.

\section{Conflicto de intereses}

Los autores declaran que no tienen conflicto de intereses.

\section{Bibliografía}

1. WHO. Coronavirus disease 2019 (COVID-19) situation report. Data as reported by national authorities by 10:00 CET 30 March 2020. Disponible en: https://www.who.int/docs/default-source/coronaviruse/situation-reports/20200330-sitrep-70-covid-19.pdf?sfvrsn=7e0fe3f8_4

2. WHO. Coronavirus disease (COVID-19) situation report - 192. Data as received by WHO from national authorities by 10:00 CEST, 30 July 2020. Disponible en: https://www.who.int/docs/default-source/coronaviruse/situation-reports/20200730-covid-19-sitrep-192.pdf?sfvrsn=5e52901f_8

3. Aguilar-Gamboa FR. Desafíos para el manejo y detección de pacientes con COVID-19 en Latinoamérica. Rev Exp Med. 2020;6(1):63-7.

4. ONU. El impacto del COVID-19 en América Latina y el Caribe. Informe junio 2020:1-29.

5. GBD 2017 Incidence and Injury Incidence and Prevalence Collaborators. Global, regional, and national incidence, prevalence, and years lived with disability for 354 diseases and injuries for 195 countries and territories, 1990-2017: a systematic analysis for the Global Burden of Disease Study. Lancet. 2018;392-1789-1858.

6. Méndez-Durán A, Méndez-Bueno H, Tapia-Yáñez T, Muñoz A, Aguilar-Sánchez L. Epidemiología de la insuficiencia renal crónica en México. Diálisis y Trasplante. 2010;31(1):7-11.

7. González MC, Rosa-Diez G, Ferreiro A. El Registro Latinoamericano de Diálisis y Trasplante Renal: la importancia del desarrollo de los registros nacionales en Latinoamérica. Nefrología Latinoamericana. 2017;14(1):12-21.

8. Sociedad Española de Nefrología. Registro SEN COVID-19. Informe 12 (18 marzo-13 de junio). Nefrología. P1-6. Disponible en: https://mailchi. $\mathrm{mp} /$ senefro/registro-epidemiolgico-vhc-vhb-vih-1314661.

9. Pallarés Carratalá V. La pandemia por la COVID-19: una oportunidad para cambiar la forma de atender a nuestros pacientes. Semergen. 2020. Article published ahead: disponible en https://doi.org/10.1016/j.semerg.2020.05.002

10. Organización Panamericana de la Salud/Organización Mundial de la Salud. La OPS advierte sobre la posible escasez de sangre durante la pandemia de COVID-19. 10 de abril de 2020. https://www.paho.org/es/ noticias/10-4-2020

11. Portolés J, Quiroga B, López JM, Cases A, Martín de Francisco AL, Arias $M$, et al. Tratamiento de la anemia renal con agentes eritropoyéticos, una revisión desde la perspectiva histórica. Rev Nefrología. 2016;7(S1):1-5.

12. Herrero JA. La anemia en la patología cardiovascular de las enfermedades renales. En: Macaya C. Libro de la salud cardiovascular. Ed. Fundación BBVA, 2007:157-163.

13. Organización Panamericana de la Salud/Organización Mundial de la Salud. Alerta epidemiológica: COVID-19, complicaciones y secuelas. 12 de agosto de 2020. Washington, D.C. OPS/OMS, 2020. 